\title{
ACTIONS DE SOINS INFIRMIERS EN SOINS PRIMAIRES COMPTE TENU DES RISQUES D'HYPERTENSION PENDANT LA GROSSESSE
}

\section{RÉVISER L'ARTICLE}

ADRIÃO, Iracely Santos ${ }^{1}$, BARBOSA, Marluce Sampaio Nobre ${ }^{2}$

ADRIÃO, Iracely Santos. BARBOSA, Marluce Sampaio Nobre. Actions de soins infirmiers en soins primaires compte tenu des risques d'hypertension pendant la grossesse. Revista Científica Multidisciplinar Núcleo do Conhecimento. An 06, Ed. 09, Vol. 04, p. 84 à 100. Septembre 2021. ISSN: 2448-0959, Lien d'accès: https://www.nucleodoconhecimento.com.br/sante/risques-dhypertension, DOI: 10.32749/nucleodoconhecimento.com.br/sante/risques-dhypertension

\section{RÉSUMÉ}

La prééclampsie se présente comme l'un des syndromes hypertenseurs qui peuvent affecter les femmes pendant la grossesse, pendant l'accouchement et/ou dans la période post-partum jusqu'à 10 jours, de sorte qu'il s'agit toujours d'une pathologie qui, lorsqu'elle n'est pas correctement diagnostiquée et traitée, peut causer de grands dommages et même entraîner la mort de la femme enceinte et/ou parturiente. L'objectif principal de cette étude est de connaître la prévention de la prééclampsie en soins primaires par les soins infirmiers. Par conséquent, la question suivante est: quelles sont les actions infirmières en soins primaires face aux risques d'hypertension pendant la grossesse? Le présent travail est basé sur une recherche bibliographique, avec une approche qualitative réalisée à travers la méthode de la revue intégrative. Pour collecter les données, une enquête a été menée dans les bases de données: Google Scholar, Pubmed, Scielo et Medline. Les descripteurs utilisés étaient les suivantes: Risques. Gestation. Hypertension. Allaitement. Les critères d'inclusion utilisés pour la sélection de l'échantillon étaient les suivants: articles disponibles publiés électroniquement de 2015 à 2020; langue portugaise. Les critères d'exclusion

\footnotetext{
1 Étudiante en sciences infirmières à l'Université CEUMA.

${ }^{2}$ Master en maladies tropicales.
}

RC: 99508

Lien d'accès: https://www.nucleodoconhecimento.com.br/sante/risques-dhypertension 
étaient les articles inférieurs à 2015 et les mots-clés qui ne sont pas liés au thème. Pour l'analyse complète des articles sélectionnés, une plate-forme en ligne (recherche dans les bases de données) a été utilisée, dans le but d'extraire (titre et objet des articles), d'organiser (sous forme de tableau), de résumer les informations et de faciliter la formation de la base de données. Les résultats ont indiqué que 35 articles ont été publiés, mais seulement 15 articles, au cours des cinq dernières années, ont pour thème principal la prévention et les risques de prééclampsie dans les soins primaires. II est conclu que les principales conduites utilisées non seulement par les infirmières, mais par toute l'équipe travaillant dans les soins primaires, devraient être basées sur le processus d'accueil axé sur les actions qui peuvent être déterminantes dans la prévention de la prééclampsie.

Mots-clés: Risques, Grossesse, Hypertension, Infirmière.

\section{INTRODUCTION}

Nous vivons actuellement dans un monde profondément marqué par la production de connaissances dans tous les domaines, y compris les soins infirmiers et la médecine. Même avec cela, le nombre de femmes touchées par des maladies largement connues, telles que le syndrome de grossesse hypertensive spécifique (SHEG), est encore très important. Son incidence est élevée, au point d'être traitée comme un problème de santé publique (ARAÚJO, et al, 2017).

Si les syndromes hypertenseurs liés à l'état de grossesse des femmes sont si importants, ils doivent donc être connus des autorités et des professionnels de la santé, et il faut donc se le demander, car au 21ème siècle, de telles pathologies compromettent encore la santé et souvent même la vie des femmes enceintes et des nouveau-nés. Lorsqu'on sait que la pratique des examens prénataux permettrait d'identifier le problème, ou du moins de permettre son diagnostic précoce, ce qui en pratique permettrait une intervention rapide (ARNALDO; CARDOZO, 2021).

Toutes ces réflexions ont contribué et motivé le choix du thème comme objet d'étude, car sa faisabilité était en partie le problème suivant: il y a eu un taux élevé de femmes

RC: 99508

Lien d'accès: https://www.nucleodoconhecimento.com.br/sante/risques-dhypertension 
enceintes qui sont presque toujours prises en charge pour présenter des signes et des symptômes de SHEG, en particulier la prééclampsie. Alors, quelles sont les actions d'allaitement en soins primaires compte tenu des risques d'hypertension pendant la grossesse? Et comme réponse provisoire au problème énoncé, il a été adopté I'hypothèse que les femmes en période gestationnelle et même parturiente et dans la période post-partum, présentent un indice de prééclampsie élevé, parce qu'elles n’avaient pas un suivi prénatal adéquat (ARAÚJO et al., 2017).

Les études impliquant cet ensemble d'informations (risques d'hypertension pendant la grossesse), aident à la prévention de l'hypertension, car elles stimulent et alertent la population à adopter des changements dans les habitudes alimentaires et dans la pratique habituelle de l'activité physique, ainsi que facilitent l'approche médicamenteuse de ses composants isolés ou syndrome métabolique lui-même, par le biais de professionnels et de chercheurs (ARNALDO; CARDOZO, 2021).

Le choix du thème agit pour apporter aux femmes enceintes des informations qui les mènent à une vie plus saine, car c'est la tâche principale des professionnels de la santé. Et pour ces femmes en grossesse devrait commencer à prendre à leur jour à leur jour une alimentation plus saine et naturelle, ayant comme conseil la consommation de fruits, légumes, légumes. Être orienté pour minimiser la consommation de viande, de graisses, d'aliments frits, de sandwichs et d'autres formes d'aliments nocifs pour la santé et qui conduit à une hypertension accrue (SOARES et al., 2015).

Pour une bonne qualité de vie, la femme enceinte considérée comme hypertensive doit maintenir une vie saine, car beaucoup d'entre eux adhèrent au traitement pharmacologique, où la pression artérielle est contrôlée par l'ingestion de comprimés. Il existe également un traitement non pharmacologique qui est effectué par le biais de pratiques d'activité physique, qui peut également être un complément au traitement pharmacologique (MELO et al., 2015).

La pratique de l'exercice est d'une importance fondamentale pour la santé et la qualité de vie en général et en particulier pour les femmes enceintes, qui ont l'habitude de

RC: 99508

Lien d'accès: https://www.nucleodoconhecimento.com.br/sante/risques-dhypertension 
mener une vie sédentaire, car elles sont dans la période de repos de la profession et commencent un mode de vie sédentaire (SOARES et al., 2015).

Le travail est justifié parce qu'il est d'un grand intérêt pour les infirmières et la femme qui a l'intention d'être mère. L'intérêt pour la recherche a également été motivé par le fait qu'il s'agit d'un thème qui apporte des informations importantes et fondamentales à chaque femme qui vise à devenir enceinte et pour le domaine d'activité des soins infirmiers.

Et donc la recherche apporte des enseignements à une bonne qualité de vie des femmes enceintes, puisque les femmes hypertensives doivent maintenir une vie saine, car beaucoup d'entre elles adhèrent à un traitement pharmacologique, où la pression artérielle est contrôlée par l'ingestion de comprimés. II existe également un traitement non pharmacologique qui est effectué par le biais de pratiques d'activité physique, qui peuvent également être un complément au traitement pharmacologique (SANTOS; NETO, 2016).

L'objectif principal de ce travail est de connaître les conduites de prévention de la prééclampsie dans les soins primaires par les soins infirmiers.

\section{MÉTHODOLOGIE}

En ce qui concerne les procédures méthodologiques de la présente étude, le développement de la recherche bibliographique a été utilisé, c'est-à-dire une revue de la littérature des articles scientifiques sur l'hypertension et la grossesse. Pour la présente recherche scientifique, les articles ont été recherchés dans les bases de données de Google Scholar, Pubmed, Scielo et Medline, des livres de revues de santé et des articles scientifiques, basés sur des documents déjà élaborés.

En outre, la recherche exploratoire pour Lakatos et Marconi (2010, p. 171) « les études exploratoires-descriptives combinées ont pour objectifs de décrire un phénomène complètement certain, tel que l'étude d'un cas pour lequel des analyses empiriques et théoriques sont effectuées ». 
Par conséquent, la recherche cherche toujours la meilleure approximation du chercheur avec la situation réelle des problèmes, en outre, elle consiste à comprendre de manière moins totalitaire, à fragmenter les opinions présentées et à viser la perspective de la perspective des acteurs impliqués dans le processus.

En ce qui concerne les moyens, les procédures de recherche bibliographique ont été appliquées à cette étude. Ainsi, des publications nationales, dans des livres, des articles rédigés par des professionnels des soins infirmiers et des revues feront partie de la construction de ce matériel (FONSECA, 2002).

Par conséquent, il est à noter que, malgré tous les efforts déployés pour élever les points de vue des acteurs sociaux impliqués dans le processus auquel la recherche est proposée d'étudier, il est perçu que la base théorique par le biais de l'enquête bibliographique devient le fondement de la compréhension de la recherche.

Dans cette étude, la compréhension de certains auteurs a été soulevée sur la base de recherches bibliographiques en détail à la recherche du point de vue de cette étude. Enrichir en connaissances, car il est d'une importance fondamentale de vérifier la position de ces auteurs concernant l'allaitement et les risques d'hypertension pendant la grossesse.

Par conséquent, les critères d'inclusion étaient: les articles publiés en portugais, qui traitent de l'allaitement et des risques d'hypertension pendant la grossesse, et qui ont été publiés et indexés dans ces bases de données de 2015 à 2020. Les critères d'exclusion étaient les articles inférieurs à 2015 et les mots-clés qui ne sont pas liés au thème. Les mots clés utilisés étaient: Risques. Gestation. Hypertension. Allaitement. La collecte de données a également été effectuée au cours du premier semestre de 2021.

La présente recherche avait parmi ses sources de collecte de données, par le catalogage et le reliure de la documentation bibliographique, des livres, des articles, des magazines, des journaux, des périodiques et des articles publiés sur Internet, d'auteurs contemporains, à travers lesquels plusieurs interprétations du thème ont été effectuées. Ainsi, il est important de mentionner que le moyen de collecter des $\mathrm{RC}: 99508$ 
données connexes est censé cultiver de la meilleure façon possible les origines et les motivations des réponses trouvées ou non à la fin de la recherche.

\section{DÉVELOPPEMENT}

L'échantillon final de cette revue se composait de 15 (quinze) articles scientifiques, sélectionnés selon des critères d'inclusion précédemment établis. Le tableau 1 représente les spécifications de chacun des articles, réparties selon: année; périodique; nom et titre des auteurs.

Table 1. Liste des études sélectionnées pour l'année, la revue, les auteurs et le titre entre 2015 et 2020.

\begin{tabular}{|c|c|c|c|}
\hline ANNÉE & PÉRIODIQUE & AUTEURS & TITRE \\
\hline 2017 & $\begin{array}{l}\text { Rev enferm UFPE } \\
\text { on line }\end{array}$ & $\begin{array}{l}\text { ARAÚJO, Isabella } \\
\text { Félix Meira, et al }\end{array}$ & $\begin{array}{l}\text { Síndromes hipertensivas e } \\
\text { fatores de risco associados } \\
\text { à gestação }\end{array}$ \\
\hline 2021 & $\begin{array}{l}\text { Revista científica } \\
\text { multidisciplinar } \\
\text { núcleo do } \\
\text { conhecimento }\end{array}$ & $\begin{array}{l}\text { ARNALDO, Mariany } \\
\text { de } \quad \text { Freitas. } \\
\text { CARDOZO, } \\
\text { Maryanne Neuraide } \\
\text { Freire }\end{array}$ & $\begin{array}{l}\text { Assistência de enfermagem } \\
\text { às gestantes hipertensas na } \\
\text { prevenção da } \\
\text { prematuridade: revisão } \\
\text { bibliográfica }\end{array}$ \\
\hline 2019 & $\begin{array}{ll}\text { Trabalho } & \text { de } \\
\text { Conclusão } & \text { de } \\
\text { Curso } & \end{array}$ & $\begin{array}{l}\text { CABRAL, Gustavo } \\
\text { Pereira }\end{array}$ & $\begin{array}{l}\text { Cuidados de enfermagem a } \\
\text { mulheres com doença } \\
\text { hipertensiva específica da } \\
\text { gestação: uma revisão } \\
\text { integrativa }\end{array}$ \\
\hline 2017 & $\begin{array}{l}\text { International } \\
\text { Nursing Congress }\end{array}$ & $\begin{array}{l}\text { ENDRINGER, } \\
\text { Deyvid Dantas; } \\
\text { CRUZ, Monielle Lima }\end{array}$ & $\begin{array}{l}\text { Representatividade do } \\
\text { enfermeiro na assistência a } \\
\text { gestantes com pré- } \\
\text { eclâmpsia }\end{array}$ \\
\hline 2015 & $\begin{array}{lr}\text { REBES - Revista } \\
\text { Brasileira de }\end{array}$ & $\begin{array}{l}\text { MELO, Wyara } \\
\text { Ferreira, et al }\end{array}$ & $\begin{array}{l}\text { A hipertensão gestacional e } \\
\text { o risco de pré-eclampsia: }\end{array}$ \\
\hline
\end{tabular}

RC: 99508

Lien d'accès: https://www.nucleodoconhecimento.com.br/sante/risques-dhypertension 


\begin{tabular}{|c|c|c|c|}
\hline & Educação e Saúde & & revisão bibliográfica \\
\hline 2018 & $\begin{array}{l}\text { Brazilian Journal of } \\
\text { Surgery and } \\
\text { Clinical Research - } \\
\text { BJSCR }\end{array}$ & $\begin{array}{l}\text { OLIVEIRA, Leilyanne } \\
\text { de Araújo Mendes, et } \\
\text { al }\end{array}$ & $\begin{array}{lr}\text { Cuidados de enfermagem a } \\
\text { gestante com síndrome } \\
\text { hipertensiva: } & \text { revisão } \\
\text { integrativa. } & \end{array}$ \\
\hline 2018 & $\begin{array}{l}\text { Revista Científica } \\
\text { Multidisciplinar } \\
\text { Núcleo do } \\
\text { Conhecimento }\end{array}$ & $\begin{array}{l}\text { SANTOS, Keilane } \\
\text { Carvalho. } \\
\text { BRASILEIRO, } \\
\text { Marislei Espíndula }\end{array}$ & $\begin{array}{l}\text { Enfermagem e os cuidados } \\
\text { Emergenciais na doença } \\
\text { hipertensiva especifica na } \\
\text { gravidez }\end{array}$ \\
\hline 2016 & $\begin{array}{l}\text { Revista Científica } \\
\text { da FASETE }\end{array}$ & $\begin{array}{l}\text { SANTOS, Joelma } \\
\text { Oliveira Silva; NETO, } \\
\text { Thiago Paulo de } \\
\text { Almeida }\end{array}$ & $\begin{array}{l}\text { Atuação do enfermeiro na } \\
\text { redução da ocorrência da } \\
\text { pré-eclâmpsia: uma revisão } \\
\text { integrativa }\end{array}$ \\
\hline 2020 & Enfermagem Brasil & $\begin{array}{l}\text { SARMENTO, Rayani } \\
\text { Silva, et al }\end{array}$ & $\begin{array}{l}\text { Pré-eclâmpsia na gestação: } \\
\text { ênfase na assistência de } \\
\text { enfermagem }\end{array}$ \\
\hline 2016 & $\begin{array}{l}\text { Centro } \\
\text { Universitário Ritter } \\
\text { dos Reis }\end{array}$ & $\begin{array}{lr}\text { TEIXEIRA, } & \text { Rafaela } \\
\text { da Rosa; } & \text { MINUZZI, } \\
\text { Renata } & \text { Nolibus; } \\
\text { TELES, } & \text { Jéssica } \\
\text { Machado } & \end{array}$ & $\begin{array}{l}\text { Cuidados de enfermagem à } \\
\text { pacientes com hipertensão } \\
\text { gestacional: um relato de } \\
\text { experiência }\end{array}$ \\
\hline 2017 & $\begin{array}{l}\text { Rev Port Med } \\
\text { Geral Fam }\end{array}$ & $\begin{array}{l}\text { FERREIRA, Sara } \\
\text { Santos, et al }\end{array}$ & $\begin{array}{l}\text { Ácido acetilsalicílico na } \\
\text { prevenção da pré- } \\
\text { eclâmpsia: uma revisão } \\
\text { baseada na evidência }\end{array}$ \\
\hline 2015 & $\begin{array}{l}\text { Ciência } \\
\text { Enfermeria }\end{array}$ & $\begin{array}{l}\text { KRAUZER, Ivete } \\
\text { Maroso, et al. }\end{array}$ & $\begin{array}{l}\text { Sistematização da } \\
\text { assistência de enfermagem } \\
\text { na atenção básica: o que } \\
\text { dizem os enfermeiros? }\end{array}$ \\
\hline 2015 & $\begin{array}{l}\text { J. res.: fundam. } \\
\text { care. }\end{array}$ & $\begin{array}{l}\text { FERNANDES, } \\
\text { Amélia Carolina }\end{array}$ & $\begin{array}{l}\text { Sistematização da } \\
\text { assistência de enfermagem }\end{array}$ \\
\hline
\end{tabular}




\begin{tabular}{|c|c|c|c|}
\hline & & Lopes & $\begin{array}{l}\text { na prevenção de infecções } \\
\text { em unidade de terapia } \\
\text { intensiva }\end{array}$ \\
\hline 2015 & $\begin{array}{l}\text { Escola Anna Nery } \\
\text { Revista de } \\
\text { Enfermagem }\end{array}$ & $\begin{array}{l}\text { SOARES, Mirelle } \\
\text { Inácio, et al }\end{array}$ & $\begin{array}{l}\text { Sistematização da } \\
\text { assistência de enfermagem: } \\
\text { facilidades e desafios do } \\
\text { enfermeiro na gerência da } \\
\text { assistência }\end{array}$ \\
\hline 2016 & Rev. Interd & $\begin{array}{l}\text { MONTEIRO, M. M., } \\
\text { et al. }\end{array}$ & $\begin{array}{l}\text { Emergências obstétricas: } \\
\text { características de casos } \\
\text { atendidos por serviço móvel } \\
\text { de urgência }\end{array}$ \\
\hline
\end{tabular}

Source : Propre auteur (2021)

\subsection{CARACTÉRISTIQUES DE L'HYPERTENSION PENDANT LA GROSSESSE}

Dans cette recherche, nous mettrons l'accent sur une pathologie qui est la deuxième cause de décès périnatal au Brésil, qui est l'hypertension pendant la grossesse, dans laquelle elle comporte plusieurs risques pour la mère et l'enfant, il est donc nécessaire de suivre régulièrement ces patients et de guider les changements de mode de vie et de régime alimentaire (ARAÚJO et al., 2017).

La pression artérielle (PA) présente des altérations dans l'interaction des facteurs neuro humoraux, comportementaux et environnementaux. L'hypertension $(\mathrm{AH})$ peut être conceptualisée comme un syndrome d'origine multifactorielle, considéré comme l'un des problèmes majeurs dans le domaine de la santé publique faisant l'objet d'une évaluation en tant que facteur de risque grave de maladies cardiovasculaires (ARNALDO; CARDOZO, 2021).

Les femmes qui utilisent des cigarettes et des contraceptifs, âgées de plus de 30 ans, finissent par être les plus touchées, car elles sont soumises à d'autres maladies RC: 99508

Lien d'accès: https://www.nucleodoconhecimento.com.br/sante/risques-dhypertension 
pulmonaires. Les femmes reçoivent une protection en raison d'événements cardiovasculaires qui se produisent avant la ménopause, chez les hommes ne surviennent qu'après l'âge de 30 ans (SOARES et al., 2015).

Des études à plus long terme ont identifié qu'ils ne sont pas différents des hommes en ce qui concerne la réponse antihypertensive de la pression artérielle; cependant, il est d'une importance primordiale d'éviter l'utilisation d'inhibiteurs enzymatiques de l'angiotensine à l'utilisation (ECA) et d'antagonistes des récepteurs de l'angiotensine II chez les femmes en âge de procréer et qui n'utilisent pas de méthodes anticonceptes sûres et éprouvées (SANTOS; NETO, 2016).

Les causes de l'hypertension sont associées à une vie sédentaire, à une consommation insuffisante d'aliments, tels qu'un excès de sodium et de graisse ou de consommation d'alcool, et même au tabagisme, qui est la cause de l'insuffisance cardiaque. Le fait est que l'hypertension est un puissant contributeur aux maladies cardiovasculaires, attaquant et surchargeant le cœur jusqu'à ce qu'un jour il échoue (CABRAL, 2019).

L'hypertension pendant la grossesse est la deuxième cause de décès maternel et périnatal au Brésil selon les données du ministère de la Santé. Cela montre la nécessité de connaître et de contrôler cette pathologie. Pendant la grossesse, l'organisme de la femme subit plusieurs transformations pour pouvoir développer le fœtus qui sera abrité pendant environ 280 jours ou environ 9 mois. Au Brésil, la population devrait atteindre la barre des 228 millions d'habitants en 2025 (CABRAL, 2019).

Le taux de mortalité selon I'IBGE en 2016 est de 6,6 naissances vivantes pour mille habitants. Compte tenu de ces données, il est évident qu'il est nécessaire de développer la recherche et la conception pour améliorer cet indice alarmant, à partir de la cartographie des endroits qui présentent le risque gestationnel le plus élevé, ce qui peut intensifier les soins aux femmes enceintes et ainsi améliorer le taux de mortalité (ENDRINGER; CRUZ, 2017). 


\subsection{ASPECTS GÉNÉRAUX DE LA PREECLAMPSIA}

On ne sait pas avec certitude quand les premiers cas de cette pathologie ont été identifiés, mais ce que l'on sait, c'est qu'elle a été décrite depuis l'époque où les enregistrements ont commencé à être faits, son apparition a été attribuée à la mauvaise humeur, au mauvais génie et, plus récemment, à l'apport en sodium et à la prise de poids pendant la grossesse (ENDRINGER; CRUZ, 2017).

On remarque que ce syndrome a provoqué des troubles majeurs chez les jeunes femmes enceintes corrélés avec la première grossesse, et est caractérisé comme la principale cause de mortalité maternelle au Brésil. En 1998, la toxémie de grossesse était responsable de $21 \%$ des décès obstétricaux directs (SANTOS; BRASILEIRO, 2018).

La prééclampsie est caractérisée comme l'un des syndromes hypertenseurs qui peuvent affecter les femmes pendant la grossesse, pendant l'accouchement et/ou dans la période post-partum jusqu'à 10 jours, de sorte qu'il s'agit toujours d'une pathologie qui, lorsqu'elle n'est pas correctement diagnostiquée et traitée, peut causer de grands dommages et même entraîner la mort de la femme enceinte et/ou parturiente (SARMENTO et al., 2020).

Il est caractérisé comme une maladie systémique qui survient à partir de la 20ème semaine de gestation par la triade: hypertension, œdème et protéinurie et peut avoir son état aggravé pour l'éclampsie, en présence de convulsions, et/ou évoluer vers le syndrome HELLP (SANTOS; BRASILEIRO, 2018).

Les causes de la prééclampsie sont encore inconnues, cependant, ce que l'on sait, c'est que certaines femmes enceintes sont plus susceptibles d'être touchées par cette pathologie. Dans l'Antiquité, ils se réfèrent à de mauvais génies, à un gain de poids et à une consommation excessive de sel (FERREIRA et al., 2017).

Aujourd'hui, on ne sait pas encore avec certitude les raisons causales et déclenchantes de la prééclampsie, ce que l'on sait, c'est qu'elle a des multi causaux: famille de prédisposition et génétique, endocrinienne-métabolique, y compris RC: 99508

Lien d'accès: https://www.nucleodoconhecimento.com.br/sante/risques-dhypertension 
l'altération de la production de prostaglandines, l'ischémie utéro placentaire, immunologique, présence de tissu trophoblastique, toxines dans la circulation sanguine maternelle, provoquant une vasoconstriction et un placenta défectueux (SARMENTO et al., 2020).

La prééclampsie a plusieurs étiologies qui peuvent provenir de causes héréditaires et/ou acquises en raison du mode de vie du patient ou des conditions sociales et environnementales. Cependant, ce que l'on sait, c'est que les conditions sociales, associées à une vie sédentaire, à une mauvaise alimentation et au manque d'informations généralement dérivées d'une faible scolarité, favorisent le risque imminent d'apparition de la prééclampsie, en plus des autres (TEIXEIRA; MINUZZI; TELES, 2016).

Le syndrome hypertensif se présente d'abord sous la forme d'une prééclampsie légère, puis évolue vers une prééclampsie sévère, une éclampsie, un syndrome de Hellp, une CIVD (coagulation intravasculaire disséminée) et enfin la mort. Et ce sont les stades de gravité du syndrome hypertenseur, cependant, il n'a pas nécessairement besoin de passer par tous les stades, et peut évoluer d'une prééclampsie légère au syndrome de Hellp (FERREIRA et al., 2017).

Les autres complications de la prééclampsie sont: un décollement placentaire prématuré à la suite d'une lésion placentaire au site d'implantation, un œdème pulmonaire aigu, une insuffisance rénale aiguë due à une glomérulopathie installée et une hémorragie cérébrale (TEIXEIRA; MINUZZI; TELES, 2016).

Les radicaux libres $\mathrm{O} 2$ sont identifiés avec des cellules graisseuses qui sont de l'endothélium, solubles dans le sang maternel et très proches de l'endothélium vasculaire. Ils réduisent la production de prostacycline et d'oxyde nitrique qui sont des vaisseaux dilatés, et augmentent la résistance vasculaire, provoquant une augmentation de la pression artérielle (FERREIRA et al., 2017).

La seule alternative pour contrôler la prééclampsie et la prévenir de l'éclampsie est le suivi correct des soins prénataux pendant la grossesse. Les patients atteints de 
prééclampsie légère doivent opter pour le repos, toujours mesurer la fréquence de la pression artérielle et adhérer à un régime pauvre en sel (KRAUZ et al., 2015).

Les médicaments antihypertenseurs et anticonvulsivants sont indiqués pour le contrôle de l'éclampsie avec une plus grande gravité, ce qui peut nécessiter l'anticipation de l'accouchement. Et la maladie a tendance à régresser spontanément avec l'ablation du placenta (FERNANDES, 2015).

Par conséquent, selon Varella (2020, p. 1), il est important de suivre quelques recommandations:

- Se dirigir ao ginecologista antes de engravidar para uma avaliação clínica e início da administração de ácido fólico;

- Importante estar presente em todas as consultas previstas no pré-natal;

- Qualquer desatenção e a ausência de sintomas podem fazer com que uma forma leve de pré-eclâmpsia evolua com complicações;

- Realizar exercícios físicos de acordo com a fase da gestação;

- Reduzir a quantidade de sal nas refeições, não fumar e principalmente não ingerir álcool durante a gravidez (VARELLA, 2020, p. 1).

Le traitement consiste en un repos au lit (côté gauche), dans un endroit aéré et calme, avec l'utilisation de médicaments, dans le but de minimiser l'aggravation et le report du travail prématuré, lorsque cela est possible, c'est-à-dire lorsqu'il n'y a pas de risque imminent pour la mère et le fœtus. Cependant, lorsque cela n'est pas possible, l'indication est l'interruption immédiate de la grossesse, quelle que soit la période gestationnelle (FERREIRA et al., 2017).

\subsection{SOINS INFIRMIERS AU NIVEAU DES SOINS DE SANTÉ DES FEMMES}

La systématisation des soins infirmiers (SAE) vise une prise en charge large et dynamique, avec une planification impliquant des processus infirmiers dans leur ensemble, dont les soins comptent sur la participation harmonieuse de plusieurs professionnels avec la famille et le patient, en construisant un environnement propice RC: 99508

Lien d'accès: https://www.nucleodoconhecimento.com.br/sante/risques-dhypertension 
à la récupération réussie et avec moins d'usure pour le patient-famille et l'équipe (SOARES et al., 2015).

Les soins infirmiers visent également l'intégration dans la société, où leur objectif principal est d'assurer une qualité de vie aux personnes, afin de mener à une meilleure et plus large prise en charge de leurs besoins spécifiques, en donnant la priorité aux personnes qui sont sous soins infirmiers et attention, c'est-à-dire celles qui font déjà un suivi (MONTEIRO et al., 2015).

«Planned Nursing» cherche à effectuer des conseils professionnels concernant l'élaboration d'une planification systématique des soins infirmiers. La série de principes coordonnés ici est attribuée à la nomenclature du «Plan de soins infirmiers thérapeutiques », un plan qui compose l'ensemble du processus d'actions et de décisions visant à programmer les soins infirmiers. (SOARES et al., 2015, p. 50).

II est entendu que les soins infirmiers sont tous les soins ou techniques dispensés à un patient, et chaque procédure qui réduit ou minimise la douleur. Lorsque l'infirmière prend soin d'un patient, il dispense non seulement de l'attention, mais se connecte au client pour réduire sa douleur, assurant ainsi son rétablissement, réduisant ses blessures et encourageant son amélioration physique et mentale, par l'action avec l'équipe de santé et la famille (MONTEIRO, et al., 2015).

Cependant, la planification des soins infirmiers est une mission qui présente un certain degré de complexité en raison de plusieurs facteurs: l'un de ces facteurs est que le processus de planification en soins infirmiers implique la participation harmonieuse de plusieurs personnes, telles que le patient, l'infirmière et son équipe et les membres de l'équipe de santé; une autre raison est le caractère indispensable de l'utilisation du raisonnement, de la prise de décision, du rendement et de la documentation; en plus de ceux-ci, une autre raison prépondérante est liée aux changements constants dans la vie qui affectent même les plans les plus améliorés (SOARES et al., 2015).

On observe que pour effectuer de bons soins infirmiers, il est nécessaire de prendre en compte plusieurs facteurs, l'un des principaux facteurs étant la capacité des infirmières à mener un travail avec l'équipe de santé, en prenant soin des paradigmes $\mathrm{RC}: 99508$

Lien d'accès: https://www.nucleodoconhecimento.com.br/sante/risques-dhypertension 
du patient et de sa famille. Utiliser sa capacité à transmettre des informations et, d'autre part, à savoir écouter, et cela constitue un élément de base pour un bon professionnel infirmier (MONTEIRO et al., 2015).

L'infirmière soignante fait partie d'une équipe multidisciplinaire qui vise des actions préventives et curatives au sein de la communauté à travers la promotion de politiques de santé visant à réduire les notifications de dommages, à travers la connaissance des causes et des risques auxquels la population est exposée, et en même temps, la sensibilisation des organismes publics aux actions qui devraient être mises en œuvre (KRAUZER, 2015).

Lors de l'anamnèse chez un client donné, un regard réfléchi et holistique doit être adopté visant non seulement la pathologie sous-jacente ou celle qui est en évidence, mais aussi pour essayer de comprendre les raisons pour lesquelles elle est apparue, afin de rechercher une solution pour le patient (SOARES et al., 2015).

Dans cette collecte de données, les infirmières devraient élaborer des plans de soins systématisés afin de promouvoir et de retrouver la santé, qui avait été ébranlée, en vue d'un diagnostic correct. Pour Carpenito apud Fernandes, et al (2015, p. 1 581):

O diagnóstico de enfermagem baseia-se tanto nos problemas reais (voltados para o presente) quanto nos problemas potenciais (voltados para o futuro), que podem ser sintomas de disfunções fisiológicas, comportamentais, psicossociais ou espirituais.

II est entendu que le diagnostic infirmier est divisé en deux moments: un diagnostic réel qui se caractérise par les manifestations définies déjà existantes chez l'individu, c'est-à-dire une mobilité physique altérée, liée à une lésion des membres inférieurs. Le second est que le diagnostic de risque est caractérisé par des facteurs de risque auxquels l'individu est exposé, vulnérable à la fois par l'environnement et par sa propre immunité physique, c'est-à-dire par le risque d'infection lié au moment de I'hospitalisation (KRAUZER, 2015).

RC: 99508

Lien d'accès: https://www.nucleodoconhecimento.com.br/sante/risques-dhypertension 


\subsection{SOINS INFIRMIERS ET ACTIONS DE PRÉVENTION PRÉ- ÉCLAMPSIE}

Au cours des soins prénataux, certains tests sont demandés et à travers ceux-ci peuvent être détectés les pathologies aiguës ou chroniques qui sont dirigées vers le traitement afin de ne pas nuire au cycle gestationnel et de diminuer les taux de morbidité et de mortalité maternelles et infantiles (ARNALDO; CARDOZO, 2021).

La consultation prénatale doit avoir lieu au début de la grossesse, car une évaluation plus détaillée des conditions maternelles et fœtales peut être effectuée. Même si la grossesse est considérée comme un processus physiologique normal, plusieurs types de changements se produisent dans le corps de la femme, ce qui entraîne une distance plus courte entre la santé et la maladie que lorsqu'elle n'est pas enceinte. La prévention ou au moins le diagnostic précoce des signes anormaux, et immédiatement un traitement efficace, peuvent agir comme une stratégie pour minimiser divers types de complications associées à la parturition, non seulement pendant la période antepartum, mais aussi pendant le travail (ENDRINGER; CRUZ, 2017).

Il est connu que le professionnel de la santé doit être conscient de tout signe pouvant indiquer une situation qui compromet les soins prénataux, c'est pourquoi l'infirmière doit effectuer toutes les étapes, en plus de la demande de tests de laboratoire (SANTOS; BRASILEIRO, 2018).

Les soins infirmiers doivent toujours faire attention tout au long de la période gestationnelle afin que les événements imprévus ne se manifestent pas pendant cet événement. Les mesures de promotion de la santé et de prévention des risques pendant la grossesse doivent se concentrer sur le service à cette clientèle beaucoup plus spécifique qui nécessite tous les soins. Dans ce contexte, les soins prénataux, tels que recommandés par le ministère de la Santé, constituent le principal moyen de générer la sécurité que les clients et les professionnels de la santé doivent avoir sur la consolidation d'une grossesse sans risques ni complications (MONTEIRO et al., 2016).

RC: 99508

Lien d'accès: https://www.nucleodoconhecimento.com.br/sante/risques-dhypertension 
Dans ce biais, la consultation infirmière, le suivi de la femme enceinte pendant les soins prénataux, l'attention à toute manifestation clinique en dehors de la fonction planifiée et de conseil sont fondamentaux et doivent faire l'objet d'une surveillance appropriée. D'autre part, ils appellent l'attention sur les complications liées à I'hypertension artérielle, à la présence d'hémorragies, de saignements et de signes de prééclampsie et d'éclampsie, en tant que phares de complications et de mortalité plus profondes (SANTOS; BRASILEIRO, 2018).

La prééclampsie a son apparition lente et progressive avec des complications majeures pour la mère et le fœtus, il est donc nécessaire qu'il existe des soins prénataux de bonne qualité qui fournissent des actions préventives et l'identification des cas prédisposants afin de diagnostiquer le plus tôt possible, de commencer un traitement prophylactique. Les événements doivent également être consignés dans les dossiers médicaux afin que ces informations ne soient pas perdues et facilitent les soins futurs (MONTEIRO et al., 2016).

L'infirmière professionnelle doit effectuer dès la première consultation avec la femme enceinte, une anamnèse complète obtenant autant d'informations pertinentes pour sa santé actuelle et antérieure, en plus des antécédents familiaux, et pour cela demander des tests de routine, un soutien vitaminique, des conseils sur son alimentation, remplir sa carte de grossesse, vérifier son carnet de vaccination et son calendrier de prochaine consultation (ENDRINGER; CRUZ, 2017).

Les soins infirmiers pendant la période prénatale sont d'une importance primordiale car ils surveillent et identifient les changements possibles dans leur période initiale, et transmettent à une évaluation plus précise dans une unité de référence, avec cette prise de décision correcte peut faire une différence, et fournir à la femme enceinte une plus grande sécurité et caractériser un véritable soin préventif, il convient de mentionner que le professionnel infirmier est un protagoniste d'une importance incalculable dans les soins prénataux, parce que c'est précisément parce qu'il a le plus grand lien avec les femmes enceintes (SANTOS; BRASILEIRO, 2018). 


\section{CONSIDÉRATIONS FINALES}

L'étude, construite à partir d'une revue de la littérature spécialisée, a permis une analyse sur la prévention de la prééclampsie dans les soins primaires et, à cette fin, a fait un bref historique de l'incidence de cette pathologie, ainsi que de ses concepts et caractéristiques.

De plus, il convenait de décrire les contributions des soins infirmiers dans les soins primaires. Par conséquent, il est entendu que pour effectuer de bons soins infirmiers, il est nécessaire de prendre en compte plusieurs facteurs, l'un des principaux facteurs étant la capacité des infirmières à travailler avec l'équipe de santé, en prenant soin des paradigmes du patient et de sa famille. Utiliser sa capacité à transmettre des informations et d'autre part savoir écouter, et cela constitue des éléments de base pour un bon professionnel infirmier

La prééclampsie, bien qu'il s'agit d'une pathologie ou d'un syndrome pouvant affecter les femmes en état de grossesse, est toujours caractérisée comme un problème de santé publique, malgré tout le développement de la science, y compris dans le domaine des soins infirmiers et dans le domaine médical, précisément la raison déclenchantante de l'étude, quelle partie du problème proposée dans l'introduction, qui, selon les chercheurs, a été traité en profondeur, de sorte que les données obtenues étaient cohérentes et que les résultats de ceux-ci puissent être généralisés à l'ensemble de la population étudiée.

Ainsi, en utilisant des articles qui ont été trouvés se concentrant sur le thème de cette étude, il suscite la nécessité de faire de nombreuses autres publications sur la prévention de la prééclampsie. La plupart des études portent davantage sur le rôle des infirmières dans l'humanisation des contacts professionnels/utilisateurs au moment de la douleur aiguë ou chronique dans les services d'urgence que sur la prévention de la prééclampsie dans les soins primaires.

Alors, quelles sont les actions d'allaitement en soins primaires compte tenu des risques d'hypertension pendant la grossesse? En réponse au problème, il convient de

RC: 99508

Lien d'accès: https://www.nucleodoconhecimento.com.br/sante/risques-dhypertension 
noter que le professionnel infirmier travaillant dans un service de santé devrait tenir des conférences afin de sensibiliser à l'importance des soins prénataux le plus rapidement possible pour assurer une grossesse en bonne santé et une bonne évolution du concept et du travail.

En ce sens, il est conclu que les principales conduites utilisées non seulement par les infirmières, mais par toute l'équipe travaillant dans les soins primaires, devraient être centrées sur le processus d'accueil, axées sur les actions qui peuvent être déterminantes dans la prévention de la prééclampsie. Ainsi, il est extrêmement important de publier plus d'études axées sur la prévention de la prééclampsie dans les soins primaires.

\section{RÉFÉRENCES}

ARAÚJO, Isabella Félix Meira, et al. Síndromes hipertensivas e fatores de risco associados à gestação. Rev enferm UFPE on line., Recife, 11(Supl. 10):4254-62, out., 2017.

ARNALDO, Mariany de Freitas. CARDOZO, Maryanne Neuraide Freire. Assistência de enfermagem às gestantes hipertensas na prevenção da prematuridade: revisão bibliográfica. Revista científica multidisciplinar núcleo do conhecimento. Ano 06, Ed. 02, Vol. 02, pp. 108 - 125 . Fevereiro de 2021.

CABRAL, Gustavo Pereira. Cuidados de enfermagem a mulheres com doença hipertensiva específica da gestação: uma revisão integrativa. Trabalho de Conclusão de Curso. Uruguaiana, 2019.

ENDRINGER, Deyvid Dantas; CRUZ, Monielle Lima. Representatividade do enfermeiro na assistência a gestantes com pré-eclâmpsia. International Nursing Congress. May 9-12, 2017.

FERNANDES, Amélia Carolina Lopes. Sistematização da assistência de enfermagem na prevenção de infecções em unidade de terapia intensiva. J. res.: fundam. care. online 2015. out./dez. 6(4):1580-1589.

RC: 99508

Lien d'accès: https://www.nucleodoconhecimento.com.br/sante/risques-dhypertension 
FERREIRA, Sara Santos, et al. Ácido acetilsalicílico na prevenção da pré-eclâmpsia: uma revisão baseada na evidência. Rev Port Med Geral Fam 2017;33:118-32.

FONSECA, J. J. S. Metodologia da pesquisa científica. Fortaleza: UEC, 2002.

GIL, Antônio Carlos. Como elaborar projetos de pesquisa. 4. ed. São Paulo: Atlas, 2007.

KRAUZER, Ivete Maroso, et al. Sistematização da assistência de enfermagem na atenção básica: o que dizem os enfermeiros? Ciência Y Enfermeria XXI (2), 2015.

LAKATOS, Eva Maria; MARCONI, Marina de Andrade. Fundamentos da metodologia científica. 7. ed. São Paulo: Atlas, 2010.

MELO, Wyara Ferreira, et al. A hipertensão gestacional e o risco de pré-eclampsia: revisão bibliográfica. REBES - Revista Brasileira de Educação e Saúde (Pombal - PB, Brasil), v. 5, n. 3, p. 07-11, jul-set, 2015.

MONTEIRO, M. M., et al. Emergências obstétricas: características de casos atendidos por serviço móvel de urgência. Rev. Interd. v. 9, n. 2, p. 136-144, abr/mai/jun. 2016 143.

OLIVEIRA, Leilyanne de Araújo Mendes, et al. Cuidados de enfermagem a gestante com síndrome hipertensiva: revisão integrativa. Brazilian Journal of Surgery and Clinical Research - BJSCR. Vol.23,n.2,pp.159-164 (Jun - Ago 2018).

SANTOS, Keilane Carvalho. BRASILEIRO, Marislei Espíndula. Enfermagem e os cuidados Emergenciais na doença hipertensiva específica na gravidez. Revista Científica Multidisciplinar Núcleo do Conhecimento. Ano 03, Ed. 11, Vol. 08, pp. 17-26 Novembro de 2018.

SANTOS, Joelma Oliveira Silva; NETO, Thiago Paulo de Almeida. Atuação do enfermeiro na redução da ocorrência da pré-eclâmpsia: uma revisão integrativa. Revista Científica da FASETE 2016.1. 
SARMENTO, Rayani Silva, et al. Pré-eclâmpsia na gestação: ênfase na assistência de enfermagem. Enfermagem Brasil 2020;19(3):261-267.

SILVA, E.L. da; MENEZES, E.M. Metodologia da pesquisa e elaboração de dissertação. Florianópolis: Laboratório de Ensino à Distância da UFSC, 2001.

SOARES, Mirelle Inácio, et al. Sistematização da assistência de enfermagem: facilidades e desafios do enfermeiro na gerência da assistência. Escola Anna Nery Revista de Enfermagem 19(1) Jan-Mar 2015.

TEIXEIRA, Rafaela da Rosa; MINUZZI, Renata Nolibus; TELES, Jéssica Machado. Cuidados de enfermagem à pacientes com hipertensão gestacional: um relato de experiência. XII Semana de Extensão, Pesquisa e Pós-Graduação SEPesq - 24 a 28 de outubro de 2016

Publié: Août 2021.

Approuvé: Septembre 2021. 